Article

\title{
Stable and Convergent Finite Difference Schemes on NonuniformTime Meshes for Distributed-Order Diffusion Equations
}

\author{
M. Luísa Morgado ${ }^{1,2} \mathbb{D}$, Magda Rebelo ${ }^{3, *} \mathbb{D}$ and Luís L. Ferrás ${ }^{4}(\mathbb{D})$ \\ 1 Center for Computational and Stochastic Mathematics, Instituto Superior Técnico, University of Lisbon, \\ 1049-001 Lisbon, Portugal; luisam@utad.pt \\ 2 Department of Mathematics, University of Trás-os-Montes e Alto Douro, UTAD, 5001-801 Vila Real, Portugal \\ 3 Center for Mathematics and Applications (CMA), Department of Mathematics, NOVA School of Science and \\ Technology, FCT NOVA, Quinta da Torre, 2829-516 Caparica, Portugal \\ 4 Center of Mathematics (CMAT), University of Minho, Campus de Azurém, 4800-058 Guimarães, Portugal; \\ luislimafr@gmail.com \\ * Correspondence: msjr@fct.unl.pt
}

check for updates

Citation: Morgado, M.L.; Rebelo, M.; Ferrás, L.L. Stable and Convergent

Finite Difference Schemes on

NonuniformTime Meshes for

Distributed-Order Diffusion

Equations. Mathematics 2021, 9, 1975.

https://doi.org/10.3390/math9161975

Academic Editor: Mariano Torrisi

Received: 26 July 2021

Accepted: 14 August 2021

Published: 18 August 2021

Publisher's Note: MDPI stays neutral with regard to jurisdictional claims in published maps and institutional affiliations.

Copyright: (c) 2021 by the authors. Licensee MDPI, Basel, Switzerland. This article is an open access article distributed under the terms and conditions of the Creative Commons Attribution (CC BY) license (https:// creativecommons.org/licenses/by/ $4.0 /)$.

\begin{abstract}
In this work, stable and convergent numerical schemes on nonuniform time meshes are proposed, for the solution of distributed-order diffusion equations. The stability and convergence of the numerical methods are proven, and a set of numerical results illustrate that the use of particular nonuniform time meshes provides more accurate results than the use of a uniform mesh, in the case of nonsmooth solutions.
\end{abstract}

Keywords: distributed-order derivatives; finite differences; diffusion equations; nonuniform meshes; stability; convergence

MSC: 35R11; 65R20

\section{Introduction}

Distributed-order differential equations have been investigated intensively in the last few decades [1-7]. The concept of the distributed-order derivative was developed by Caputo [1] and further developed by Caputo, Bagley, Torvik, Mainardi, and Gorenflo (just to name a few) [2-8].

Diffusion equations with distributed-order may be regarded as a generalization of integer- and fractional-order diffusion equations, and they can involve distributed-order derivatives in time, or in space, or both. In particular, Caputo time distributed-order diffusion equations, which are useful to describe ultra-slow diffusion processes [8], are given by:

$$
\int_{0}^{1} c(\alpha) \frac{\partial^{\alpha} u(t, x)}{\partial t^{\alpha}} d \alpha=\frac{\partial^{2} u(t, x)}{\partial x^{2}}+f(t, x), 0<t \leq T, 0 \leq x \leq L,
$$

where the weighting function of the orders of the time derivatives is such that $c(\alpha) \geq 0$ and $\int_{0}^{1} c(\alpha) d \alpha=C>0[6,7]$. The fractional derivatives correspond to the Caputo derivatives, which for orders between zero and one are given by:

$$
\frac{\partial^{\alpha} u(t, x)}{\partial t^{\alpha}}=\frac{1}{\Gamma(1-\alpha)} \int_{0}^{t}(t-s)^{-\alpha} \frac{\partial u(s, x)}{\partial s} d s .
$$


In fact, we easily see that if $c(\alpha)=\delta(\alpha-\beta)$, then (1) becomes the singular-order differential equation:

$$
\frac{\partial^{\beta} u(t, x)}{\partial t^{\beta}}=\frac{\partial^{2} u(t, x)}{\partial x^{2}}+f(t, x), 0<t \leq T, 0 \leq x \leq L .
$$

When $\beta=1$, Equation (3) reduces to the classical (integer-order) diffusion equation. In the case where $0<\beta<1$, (3) is called the subdiffusion equation, and it has been the subject of investigation by many researchers.

With the growing interest on this type of equation, numerical methods started being developed. Diethelm and Ford [9] developed a basic framework for the numerical solution of distributed-order differential equations. Ford and Morgado [10] discussed the existence and uniqueness of solutions for this type of equation and also proposed a numerical method for their approximation in the case where the initial conditions are not known. Morgado and Rebelo [11] developed an implicit finite difference scheme for the numerical approximation of the distributed-order time-fractional reaction-diffusion equation with a nonlinear source term. Ye et al. [12] developed a numerical method based on a compact difference scheme for a distributed-order time-fractional diffusion-wave equation. Wang et al. [13] derived and analyzed a second-order accurate implicit numerical method for the Riesz space distributedorder advection-dispersion equation, and Jin et al. [14] presented a numerical solution of an initial boundary value problem for the distributed-order time-fractional diffusion equation. They developed a space semidiscrete scheme based on the standard Galerkin finite element method and established error estimates for both smooth and nonsmooth initial data. A survey on numerical methods for these types of equations can be found in [15].

These works struggled with the low convergence order of the numerical schemes, since the solution of fractional differential equations may be nonsmooth at $t=0$, even if the data are infinitely smooth.

In [16], the authors showed that typical solutions of the single-order differential Equation (3) possess a singularity at the origin in time, in the sense that they satisfy:

$$
\left|\frac{\partial^{\ell} u(t, x)}{\partial t^{\ell}}\right| \leq C\left(1+t^{\beta-\ell}\right), \ell=1,2, t \in(0, T],
$$

for some positive constant $C$, a fact that brings serious difficulties in the derivation of accurate numerical methods. In order to overcome this problem, the authors proposed the use of a time-graded mesh:

$$
\left\{t_{j}=T\left(\frac{j}{n}\right)^{r}\right\}_{j=0}^{n}
$$

with some grading exponent $r \geq 1$ (note that $r=1$ corresponds to a uniform mesh), in which the grading exponent is properly chosen to recover the optimal convergence order attained for sufficiently smooth solutions. Naturally, the grading exponent depends on the order of the time derivative $\beta$, and we refer the interested reader to [16] for more details.

Estimates as (4) are not easy to achieve for distributed-order diffusion equations as (1) because the order of the time derivative is distributed over the interval $[0,1]$, and to the best of our knowledge, they have not been obtained so far. The use of time-graded meshes without knowing how to properly choose the grading exponent is risky since, as explained also in [16], larger grading exponents will densely place more mesh points near the singularity, which originates the appearance of round-off errors.

Hence, in this paper, we consider initial-boundary value problems for (1), with the initial condition and boundary conditions of the form:

$$
\begin{gathered}
u(0, x)=g(x), \\
u(t, 0)=u_{0}, u(t, L)=u_{L},
\end{gathered}
$$


where we assume that all the involved functions are continuous in their respective domains.

We propose a finite difference scheme on a nonuniform time mesh:

$$
\Lambda_{t}=\left\{0=t_{0}<t_{1}<\cdots<t_{n}=T, \quad t_{i+1}=t_{i}+\tau_{i}, i=0,1, \ldots, n-1\right\} .
$$

Since a singularity at the origin in time is expected, we consider nonuniform meshes such that $\tau_{i} \leq \tau_{i+1}$. In addition to the graded meshes (5), we are also interested in nonuniform meshes of the kind (see [17]):

$$
\tau_{i}=t_{i+1}-t_{i}=(i+1)^{\gamma} \eta, i=0,1, \ldots, n-1
$$

where $\gamma$ is a positive integer and $\eta>0$.

Taking into account the summation formulas $\sum_{k=1}^{n} k=\frac{n(n+1)}{2}, \sum_{k=1}^{n} k^{2}=\frac{n(n+1)(2 n+1)}{6}$, $\sum_{k=1}^{n} k^{3}=\frac{n^{2}(n+1)^{2}}{4}$, and $\sum_{k=1}^{n} k^{4}=\frac{n(n+1)(2 n+1)\left(3 n^{2}+3 n-1\right)}{30}$, we obtain, respectively:

$$
\begin{aligned}
& \underline{\text { Mesh 1: }}(\gamma=1) \eta=\frac{2 T}{n(n+1)} ; \\
& \underline{\text { Mesh 2 }}(\gamma=2) \eta=\frac{6 T}{n(n+1)(2 n+1)} ; \\
& \underline{\text { Mesh 3: }}(\gamma=3) \eta=\frac{4 T}{n^{2}(n+1)^{2}} ; \\
& \underline{\text { Mesh 4: }}(\gamma=4) \eta=\frac{30 T}{n(n+1)(2 n+1)\left(3 n^{2}+3 n-1\right)}
\end{aligned}
$$

The paper is organized as follows. In Section 2, we describe the numerical method. In Section 3, we prove the stability and convergence of the numerical method. In Section 4, we present some numerical results that validate the theoretical results obtained in the previous section. The paper ends with the conclusions and plans for further investigation.

To the best of our knowledge, the use of this type of nonuniform meshes has never been analyzed for distributed-order diffusion equations. As we will see in the section on the numerical results, these meshes allow us to recover the optimal time convergence in the case of problems with nonsmooth solutions.

\section{Finite Difference Scheme}

In order to numerically solve the problem (1), (6), (7), we start with the discretization of the integral in (1). Let us consider a partition of the interval $[0,1]$ into $N$ subintervals, with equal length $h=1 / N$. Defining $\alpha_{j}=j h-\frac{h}{2}, j=1, \ldots, N$, we can use the midpoint rule to obtain:

$$
\int_{0}^{1} c(\alpha) \frac{\partial^{\alpha} u(t, x)}{\partial t^{\alpha}} d \alpha \approx h \sum_{j=1}^{N} c\left(\alpha_{j}\right) \frac{\partial^{\alpha_{j}} u(t, x)}{\partial t^{\alpha_{j}}} .
$$

In order to approximate the spatial derivative, we consider a uniform mesh, on the interval $[0, L]$, defined by the grid points $x_{i}=i \Delta x, i=0,1, \ldots, K$, where $\Delta x=\frac{L}{K}$, and use:

$$
\frac{\partial^{2} u\left(t, x_{i}\right)}{\partial x^{2}} \approx \frac{u\left(t, x_{i+1}\right)-2 u\left(t, x_{i}\right)+u\left(t, x_{i-1}\right)}{(\Delta x)^{2}}
$$

Substituting (14) and (15) in (1) and denoting $U_{i}(t) \approx u\left(t, x_{i}\right)$, we obtain the semidiscretized scheme:

$$
h \sum_{j=1}^{N} c\left(\alpha_{j}\right) \frac{\partial^{\alpha_{j}} U_{i}(t)}{\partial t^{\alpha_{j}}}=\frac{U_{i+1}(t)-2 U_{i}(t)+U_{i-1}(t)}{(\Delta x)^{2}}+f\left(t, x_{i}\right), \quad i=1, \ldots, K-1 .
$$


From (7), we have:

$$
U_{0}(t)=u_{0}, \quad \text { and } \quad U_{K}(t)=u_{L}
$$

and from (6):

$$
U_{i}(0)=g\left(x_{i}\right), \quad i=1, \ldots, K-1 .
$$

For the discretization of the fractional derivatives, we consider the nonuniform meshes (5) and (10)-(13) and we use the L1 approximation formula (see [18]):

$$
\frac{\partial^{\alpha_{j}} u\left(t_{k} \cdot\right)}{\partial t^{\alpha_{j}}} \approx \sum_{m=0}^{k-1} \tau_{m}^{-1} a_{m, k}^{\alpha_{j}}\left(u\left(t_{m+1}, \cdot\right)-u\left(t_{m}, \cdot\right)\right), \quad m=0,1, \ldots, k-1, k=1, \ldots, n
$$

where the coefficients $a_{m, k}^{\alpha_{j}}$ are defined by:

$$
a_{m, k}^{\alpha_{j}}=\frac{\left(t_{k}-t_{m}\right)^{1-\alpha_{j}}-\left(t_{k}-t_{m+1}\right)^{1-\alpha_{j}}}{\Gamma\left(2-\alpha_{j}\right)} .
$$

Substituting in (16) and denoting by $U_{i}^{k} \approx u\left(t_{k}, x_{i}\right)$, we obtain the finite difference scheme:

$$
\begin{aligned}
& \left.h \sum_{j=1}^{N} c\left(\alpha_{j}\right) \sum_{m=0}^{k-1} \tau_{m}^{-1} a_{m, k}^{\alpha_{j}}\left(U_{i}^{m+1}-U_{i}^{m}\right)\right)= \\
& \frac{U_{i+1}^{k}-2 U_{i}^{k}+U_{i-1}^{k}}{(\Delta x)^{2}}+f\left(t_{k}, x_{i}\right), i=1, \ldots, K-1, k=1,2, \ldots, n .
\end{aligned}
$$

Hence, in order to obtain a numerical solution for (1), (6), and (7), we need to solve the linear systems of Equation (21) noting that, taking (17) and (18) into account, we have:

$$
\begin{aligned}
& U_{0}^{l}=u_{0}, \quad U_{L}^{l}=u_{L}, \quad l=1,2, \ldots, n \\
& U_{i}^{0}=g\left(x_{i}\right), \quad i=1, \ldots, K-1 .
\end{aligned}
$$

\section{Stability and Convergence of the Numerical Method}

In this section, we analyze the stability and convergence of the numerical scheme (21)(23). In what follows, $C$, depending on the context, denotes different positive constants.

\subsection{Stability}

Let us start by defining the operators $L_{1}\left(U_{i}^{k}\right)$ and $L_{2}\left(U_{i}^{k-1}\right)$ for $i=1, \ldots, K-1, k=$ $1,2, \ldots, n$ by:

$$
\begin{gathered}
L_{1}\left(U_{i}^{k}\right)=h \sum_{j=1}^{N} c\left(\alpha_{j}\right) \tau_{k-1}^{-1} a_{k-1, k}^{\alpha_{j}} U_{i}^{k}-\frac{U_{i+1}^{k}-2 U_{i}^{k}+U_{i-1}^{k}}{(\Delta x)^{2}}, \\
L_{2}\left(U_{i}^{k-1}\right)=h \sum_{j=1}^{N} c\left(\alpha_{j}\right) \tau_{k-1}^{-1} a_{k-1, k}^{\alpha_{j}} U_{i}^{k-1}-h \sum_{j=1}^{N} c\left(\alpha_{j}\right) \sum_{m=0}^{k-2} \tau_{m}^{-1} a_{m, k}^{\alpha_{j}}\left(U_{i}^{m+1}-U_{i}^{m}\right) .
\end{gathered}
$$

Then, the numerical scheme (21)-(23) can be rewritten as:

$$
L_{1}\left(U_{i}^{k}\right)=L_{2}\left(U_{i}^{k-1}\right)+f\left(t_{k}, x_{i}\right), i=1, \ldots, K-1, k=1,2, \ldots, n .
$$

Proposition 1. The coefficients $a_{m, k^{\prime}}^{\alpha_{j}}$ for $j=1, \ldots, N$, satisfy:

(i) $\quad a_{m, k}^{\alpha_{j}}>0, m=1, \ldots, k-1, k=1,2, \ldots, n$;

(ii) $\tau_{m+1}^{-1} a_{m+1, k}^{\alpha_{j}}-\tau_{m}^{-1} a_{m, k}^{\alpha_{j}}>0, m=1, \ldots, k-1, k=1,2, \ldots, n$.

Proof. Let $j=1, \ldots, N$. 
(i) This is straightforward since $t_{m}<t_{m+1} \leq t_{k}$;

(ii) Regarding (ii), note that:

$$
\Gamma\left(2-\alpha_{j}\right) \tau_{m}^{-1} a_{m, k}^{\alpha_{j}}=\frac{\left(t_{k}-t_{m}\right)^{1-\alpha_{j}}-\left(t_{k}-t_{m+1}\right)^{1-\alpha_{j}}}{t_{m+1}-t_{m}} .
$$

By the mean value theorem, there must exist a $\xi_{1} \in\left(t_{m}, t_{m+1}\right)$ such that:

$$
\Gamma\left(2-\alpha_{j}\right) \tau_{m}^{-1} a_{m, k}^{\alpha_{j}}=-\left(1-\alpha_{j}\right)\left(t_{k}-\xi_{1}\right)^{-\alpha_{j}} .
$$

Analogously, there must exist a $\xi_{2} \in\left(t_{m+1, m+2}\right)$ such that:

$$
\Gamma\left(2-\alpha_{j}\right) \tau_{m+1}^{-1} a_{m+1, k}^{\alpha_{j}}=-\left(1-\alpha_{j}\right)\left(t_{k}-\xi_{2}\right)^{-\alpha_{j}} .
$$

Then,

$$
\begin{aligned}
& \Gamma\left(2-\alpha_{j}\right)\left(\tau_{m+1}^{-1} a_{m+1, k}^{\alpha_{j}}-\tau_{m}^{-1} a_{m, k}^{\alpha_{j}}\right)=\left(1-\alpha_{j}\right)\left(-\left(t_{k}-\xi_{2}\right)^{-\alpha_{j}}+\left(t_{k}-\xi_{1}\right)^{-\alpha_{j}}\right)>0, \\
& \text { since } \xi_{1}<\xi_{2} .
\end{aligned}
$$

The result concerning the stability of the numerical scheme is presented in the following theorem, where we prove that the error does not increase in each iteration given a small perturbation on the initial condition.

Theorem 1. The numerical scheme (21)-(23) is unconditionally stable.

Proof. In order to analyze the stability of the numerical scheme, let:

$$
\tilde{g}\left(x_{i}\right)=g\left(x_{i}\right)+\varepsilon_{i}^{0}, \quad i=1, \ldots, K-1,
$$

and let $U_{i}^{k}$ and $\tilde{U}_{i}^{k}$ be the numerical solutions corresponding to the initial data $g$ and $\tilde{g}$, respectively. We easily see that:

$$
L_{1}\left(\varepsilon_{i}^{k}\right)=L_{2}\left(\varepsilon_{i}^{k-1}\right), i=1, \ldots, K-1, k=1, \ldots, n .
$$

Define $E^{k}=\left(\begin{array}{lll}\varepsilon_{1}^{k} & \varepsilon_{2}^{k} \ldots \varepsilon_{K-1}^{k}\end{array}\right)$. We use mathematical induction to prove that:

$$
\left\|E^{k}\right\|_{\infty}=\max _{1 \leq i \leq K-1}\left|\varepsilon_{i}^{k}\right| \leq\left\|E^{0}\right\|_{\infty}, k=1, \ldots, n .
$$

Define:

$$
\Lambda\left(h, \tau_{k}\right)=h \sum_{j=1}^{N} c\left(\alpha_{j}\right) \tau_{k}^{-1} a_{k, k+1}^{\alpha_{j}}, k=0,1, \ldots, n-1
$$

Since the weighting function $c(\cdot)$ is nonnegative and $a_{k, k+1}^{\alpha_{j}}>0$ for $k=0,1, \ldots, n-1$, we have that,

$$
\Lambda\left(h, \tau_{k}\right)>0, k=0,1, \ldots, n-1 .
$$


Let us first prove that (31) holds for $k=1$. Since $\Lambda\left(h, \tau_{0}\right)\left\|E^{1}\right\|_{\infty}=\Lambda\left(h, \tau_{0}\right)\left|\varepsilon_{p}^{1}\right|$ for some $p \in\{1,2, \ldots, K-1\}$, then:

$$
\begin{aligned}
\Lambda\left(h, \tau_{0}\right)|| E^{1} \|_{\infty} & =\Lambda\left(h, \tau_{0}\right)\left|\varepsilon_{p}^{1}\right|+\frac{2\left|\varepsilon_{p}^{1}\right|-2\left|\varepsilon_{p}^{1}\right|}{(\Delta x)^{2}} \\
& \leq \Lambda\left(h, \tau_{0}\right)\left|\varepsilon_{p}^{1}\right|+\frac{2\left|\varepsilon_{p}^{1}\right|-\left|\varepsilon_{p+1}^{1}\right|-\left|\varepsilon_{p-1}^{1}\right|}{(\Delta x)^{2}} \\
& \leq\left|\Lambda\left(h, \tau_{0}\right) \varepsilon_{p}^{1}-\frac{\varepsilon_{p+1}^{1}-2 \varepsilon_{p}^{1}+\varepsilon_{p-1}^{1}}{(\Delta x)^{2}}\right| \\
& =\left|L_{1}\left(\varepsilon_{p}^{1}\right)\right|=\left|L_{1}\left(\varepsilon_{p}^{0}\right)\right|=\left|\Lambda\left(h, \tau_{0}\right)\right|\left|\varepsilon_{p}^{0}\right| \mid \\
& \leq \Lambda\left(h, \tau_{0}\right)|| E^{0} \|_{\infty}
\end{aligned}
$$

and therefore, (31) is satisfied for $k=1$.

Let us now assume that $\left\|E^{m}\right\|_{\infty} \leq\left\|E^{0}\right\|_{\infty}$ for $m=1,2, \ldots, k-1$, and let $p \in \mathbb{N}$ be such that:

$$
\left|\varepsilon_{p}^{k}\right|=\max _{1 \leq i \leq K-1}\left|\varepsilon_{i}^{k}\right|=|| E^{k} \|_{\infty}
$$

Proceeding similarly as above:

$$
\begin{aligned}
\Lambda\left(h, \tau_{k-1}\right)\left\|E^{k}\right\|_{\infty} & \leq\left|\Lambda\left(h, \tau_{k-1}\right) \varepsilon_{p}^{k}-\frac{\varepsilon_{p+1}^{k}-2 \varepsilon_{p}^{k}-\varepsilon_{p-1}^{k}}{(\Delta x)^{2}}\right| \\
& =\left|L_{1}\left(\varepsilon_{p}^{k}\right)\right|=\left|L_{2}\left(\varepsilon_{p}^{k-1}\right)\right| \\
& =\left|h \sum_{j=1}^{N} c\left(\alpha_{j}\right) \tau_{k-1}^{-1} a_{k-1, k}^{\alpha_{j}} \varepsilon_{p}^{k-1}-h \sum_{j=1}^{N} c\left(\alpha_{j}\right) \sum_{m=0}^{k-2} \tau_{m}^{-1} a_{m, k}^{\alpha_{j}}\left(\varepsilon_{p}^{m+1}-\varepsilon_{p}^{m}\right)\right| \\
& =\left|h \sum_{j=1}^{N} c\left(\alpha_{j}\right) \tau_{0}^{-1} a_{0, k}^{\alpha_{j}} \varepsilon_{p}^{0}+h \sum_{j=1}^{N} c\left(\alpha_{j}\right) \sum_{m=0}^{k-2}\left(\tau_{m+1}^{-1} a_{m+1, k}^{\alpha_{j}}-\tau_{m}^{-1} a_{m, k}^{\alpha_{j}}\right) \varepsilon_{p}^{m+1}\right| \\
& \leq h \sum_{j=1}^{N} c\left(\alpha_{j}\right) \tau_{0}^{-1} a_{0, k}^{\alpha_{j}}\left|\varepsilon_{p}^{0}\right|+h \sum_{j=1}^{N} c\left(\alpha_{j}\right) \sum_{m=0}^{k-2}\left(\tau_{m+1}^{-1} a_{m+1, k}^{\alpha_{j}}-\tau_{m}^{-1} a_{m, k}^{\alpha_{j}}\right)\left|\varepsilon_{p}^{m+1}\right|
\end{aligned}
$$

since $\left|\varepsilon_{p}^{0}\right| \leq\left\|E^{0}\right\|_{\infty}$ and $\left|\varepsilon_{p}^{m+1}\right| \leq\left\|E^{0}\right\|_{\infty}$, we have that:

$$
\begin{aligned}
\Lambda\left(h, \tau_{k-1}\right)\left\|E^{k}\right\|_{\infty} & \leq h \sum_{j=1}^{N} c\left(\alpha_{j}\right)\left(\tau_{0}^{-1} a_{0, k}^{\alpha_{j}}+\sum_{m=0}^{k-2}\left(\tau_{m+1}^{-1} a_{m+1, k}^{\alpha_{j}}-\tau_{m}^{-1} a_{m, k}^{\alpha_{j}}\right)\right)\left\|E^{0}\right\|_{\infty} \\
& =h \sum_{j=1}^{N} c\left(\alpha_{j}\right)\left(\tau_{k-1}^{-1} a_{k-1, k}^{\alpha_{j}}\right)\left\|E^{0}\right\|_{\infty} \\
& =\Lambda\left(h, \tau_{k-1}\right)\left\|E^{0}\right\|_{\infty}
\end{aligned}
$$

and the result is proven.

Note that the result concerning the stability of the numerical scheme does not depend on the definition of the mesh points (8).

\subsection{Convergence}

As in [19], we assume that that the solution of (1) with the initial condition (6) and boundary conditions (7) satisfies (4). 
In order to rewrite the L1 approximation of $\frac{\partial^{\alpha} u\left(t_{j}, \cdot\right)}{\partial t^{\alpha}}, 0<\alpha<1$, we define the operator:

$$
\delta_{t}^{\alpha} u\left(t_{j}, \cdot\right)=\sum_{m=0}^{j-1} \tau_{m}^{-1} a_{m, j}^{\alpha}\left(u\left(t_{m+1}, \cdot\right)-u\left(t_{m}, \cdot\right)\right),
$$

where $t_{j}$, given in (8), are defined according to the mesh we use.

From [19], we know that for $j=1, \ldots, n$ :

$$
\begin{aligned}
\left|\delta_{t}^{\alpha} u\left(t_{j}, \cdot\right)-\frac{\partial^{\alpha} u\left(t_{j}, \cdot\right)}{\partial t^{\alpha}}\right| & \leq t_{j}^{-\alpha}\left(\frac{\tau_{0}}{t_{j}} \Psi^{1}+\max _{k=2, \ldots, j} \Psi^{k}\right) \\
& \leq t_{j}^{-\alpha}\left(\Psi^{1}+\max _{k=2, \ldots, j} \Psi^{k}\right) \text { since } \frac{\tau_{0}}{t_{j}} \leq 1, j=1, \ldots, n,
\end{aligned}
$$

where:

$$
\begin{gathered}
\Psi^{1}=\tau_{0}^{\alpha} \sup _{s \in\left(0, t_{1}\right)}\left(s^{1-\alpha}\left|\delta_{t}^{\alpha} u\left(t_{1}, \cdot\right)-\frac{\partial u(s, \cdot)}{\partial s}\right|\right), \\
\Psi^{k}=\tau_{k-1}^{2-\alpha} t_{k}^{\alpha} \sup _{s \in\left(t_{k-1}, t_{k}\right)}\left|\frac{\partial^{2} u(s, \cdot)}{\partial s^{2}}\right|, \quad k=1,2, \ldots, n .
\end{gathered}
$$

Proposition 2. Let $\left\{t_{j}\right\}$ be the mesh points defined by $(8), \delta_{t}^{\alpha} u\left(t_{j}, \cdot\right)$ the operator defined by (33), and $u$ the solution of (1). Then, under Assumption (4), we have:

$$
\left|\delta_{t}^{\alpha} u\left(t_{j}, \cdot\right)-\frac{\partial^{\alpha} u\left(t_{j}, \cdot\right)}{\partial t^{\alpha}}\right| \leq C t_{j}^{-\alpha} n^{-\theta}, j=1, \ldots, n, \theta=\min \{a \beta, 2-\alpha\}
$$

where $a=r$ for the graded mesh (5), $a=2$ for the mesh (10), $a=3$ for the mesh (11), $a=4$ for the mesh (12), and $a=5$ for the mesh (13), and $C$ is a positive constant that does not depend on $n$.

Proof. The case where a graded mesh is used was already analyzed in [19]. Therefore, we only consider the case where the remaining nonuniform meshes are used.

Let us first analyze $\Psi^{1}$.

$$
\Psi^{1} \leq \tau_{0}^{\alpha} \sup _{s \in\left(0, t_{1}\right)}\left(s^{1-\alpha}\left|\frac{u\left(t_{1}, \cdot\right)-u\left(t_{0}, \cdot\right)}{\tau_{0}}\right|\right)+\tau_{0}^{\alpha} \sup _{s \in\left(0, t_{1}\right)}\left(s^{1-\alpha}\left|\frac{\partial u(s, \cdot)}{\partial s}\right|\right)
$$

Since:

$$
\begin{aligned}
\tau_{0}^{\alpha} \sup _{s \in\left(0, t_{1}\right)}\left(s^{1-\alpha}\left|\frac{u\left(t_{1}, \cdot\right)-u\left(t_{0}, \cdot\right)}{\tau_{0}}\right|\right) & \leq \tau_{0}^{\alpha} \tau_{0}^{-1} \tau_{0}^{1-\alpha}\left|u\left(t_{1}, \cdot\right)-u\left(t_{0}, \cdot\right)\right|=\left|\int_{0}^{t_{1}} \frac{\partial u(s, \cdot)}{\partial s} d s\right| \\
& \leq \int_{0}^{t_{1}}\left|\frac{\partial u(s, \cdot)}{\partial s}\right| d s \leq C \int_{0}^{t_{1}} s^{\beta-1} d s \\
& \leq C \tau_{0}^{\beta}
\end{aligned}
$$

and:

$$
\tau_{0}^{\alpha} \sup _{s \in\left(0, t_{1}\right)}\left(s^{1-\alpha}\left|\frac{\partial u(s, \cdot)}{\partial s}\right|\right) \leq C \sup _{s \in\left(0, t_{1}\right)} s \times s^{\beta-1}=C \tau_{0}^{\beta}
$$

we obtain:

$$
\Psi^{1} \leq C \tau_{0}^{\beta} \leq C n^{-a \beta},
$$

where $a=2$ for Mesh 1, $a=3$ for Mesh 2, $a=4$ for Mesh 3, and $a=5$ for Mesh 4 .

Let us now analyze $\Psi^{k}, k=2, \ldots, n$. 


$$
\Psi^{k} \leq C \tau_{k-1}^{2-\alpha} t_{k}^{\alpha} \sup _{s \in\left(t_{k-1}, t_{k}\right)} s^{\beta-2} \leq C \tau_{k-1}^{2-\alpha} t_{k}^{\alpha} t_{k-1}^{\beta-2}
$$

- $\quad$ For Mesh 1:

$$
\begin{aligned}
\Psi^{k} & \leq C \tau_{k-1}^{2-\alpha} t_{k}^{\alpha} t_{k-1}^{\beta-2}=C\left(k^{2} \mu\right)^{2-\alpha}\left(\frac{k(k+1)}{2} \mu\right)^{\alpha+\beta-2}\left(\frac{k+1}{k-1}\right)^{2-\beta} \\
& \leq C k^{2-\alpha+2(\alpha+\beta-2)} \mu^{\beta} \leq \tilde{C}_{2} k^{\alpha+2 \beta-2} n^{-2 \beta}=C\left(\frac{k}{n}\right)^{\alpha+2 \beta-2} n^{-(2-\alpha)} \\
& \leq C \begin{cases}n^{-2 \beta}, & \alpha+2 \beta-2<0 \\
n^{-(2-\alpha)}, & \alpha+2 \beta-2 \geq 0\end{cases}
\end{aligned}
$$

- $\quad$ For Mesh 2:

$$
\begin{aligned}
\Psi^{k} & \leq C \tau_{k-1}^{2-\alpha} t_{k}^{\alpha} t_{k-1}^{\beta-2}=C\left(k^{2} \mu\right)^{2-\alpha}\left(\frac{k(k+1)(2 k+1)}{6} \mu\right)^{\alpha+\beta-2}\left(\frac{(k+1)(2 k+1)}{(k-1)(2 k-1)}\right)^{2-\beta} \\
& \leq C k^{2(2-\alpha)+3(\alpha+\beta-2)} \mu^{\beta} \leq C k^{\alpha+3 \beta-2} n^{-3 \beta}=C\left(\frac{k}{n}\right)^{\alpha+3 \beta-2} n^{-(2-\alpha)} \\
& \leq C \begin{cases}n^{-3 \beta}, & \alpha+3 \beta-2<0 \\
n^{-(2-\alpha)}, & \alpha+3 \beta-2 \geq 0 ;\end{cases}
\end{aligned}
$$

- $\quad$ For Mesh 3:

$$
\begin{aligned}
\Psi^{k} & \leq C \tau_{k-1}^{2-\alpha} t_{k}^{\alpha} t_{k-1}^{\beta-2}=C\left(k^{3} \mu\right)^{2-\alpha}\left(\frac{(k+1)^{2} k^{2}}{4} \mu\right)^{\alpha+\beta-2}\left(\frac{k-1}{k+1}\right)^{2 \beta-4} \\
& \leq C k^{3(2-\alpha)+4(\alpha+\beta-2)} \mu^{\beta} \leq C k^{\alpha+4 \beta-2} n^{-4 \beta}=C\left(\frac{k}{n}\right)^{\alpha+4 \beta-2} n^{-(2-\alpha)} \\
& \leq C \begin{cases}n^{-4 \beta}, & \alpha+4 \beta-2<0 \\
n^{-(2-\alpha)}, & \alpha+4 \beta-2 \geq 0\end{cases}
\end{aligned}
$$

- $\quad$ For Mesh 4:

$$
\begin{aligned}
\Psi^{k} & \leq C \tau_{k-1}^{2-\alpha} t_{k}^{\alpha} t_{k-1}^{\beta-2}=C\left(k^{4} \mu\right)^{2-\alpha}\left(\frac{n(k+1)(2 k+1)\left(3 k^{2}+3 k-1\right)}{30} \mu\right)^{\alpha+\beta-2} \\
& \times\left(\frac{(k-1)(2 k-1)\left(3(k-1)^{2}+3 k-4\right)}{(k+1)(2 k+1)\left(3 k^{2}+3 k-1\right)}\right)^{\beta-2} \\
& \leq C k^{4(2-\alpha)+5(\alpha+\beta-2)} \mu^{\beta} \leq C k^{\alpha+5 \beta-2} n^{-5 \beta}=C\left(\frac{k}{n}\right)^{\alpha+5 \beta-2} n^{-(2-\alpha)} \\
& \leq C \begin{cases}n^{-5 \beta}, & \alpha+5 \beta-2<0 \\
n^{-(2-\alpha)}, & \alpha+5 \beta-2 \geq 0 .\end{cases}
\end{aligned}
$$

We may then conclude that:

$$
\left|\delta_{t}^{\alpha} u\left(t_{j}, \cdot\right)-\frac{\partial^{\alpha} u\left(t_{j}, \cdot\right)}{\partial t^{\alpha}}\right| \leq C t_{j}^{-\alpha} n^{-\theta}, \quad \theta=\min \{a \beta, 2-\alpha\},
$$

where $a=2$ for Mesh 1, $a=3$ for Mesh 2, $a=4$ for Mesh 3, and $a=5$ for Mesh 4.

In order to proceed to the error analysis, let us first note that taking into account the error committed in the discretization of the integral term in (1) and in the approximations 
of the fractional time derivatives and the second-order space derivative, the solution of (1) at $(t, x)=\left(t_{k}, x_{i}\right)$ satisfies:

$$
\begin{aligned}
& h \sum_{j=1}^{N} c\left(\alpha_{j}\right)\left(\sum_{m=0}^{k-1} \tau_{m}^{-1} a_{m, k}^{\alpha_{j}}\left(u\left(t_{m+1}, x_{i}\right)-u\left(t_{m}, x_{i}\right)\right)-\left(\delta_{t}^{\alpha_{j}} u\left(t_{k}, x_{i}\right)-\frac{\partial^{\alpha_{j}} u\left(t_{k}, x_{i}\right)}{\partial t^{\alpha_{j}}}\right)\right)+\frac{h^{2}}{24} H^{\prime \prime}(v) \\
& =\frac{u\left(t_{k}, x_{i+1}\right)-2 u\left(t_{k}, x_{i}\right)+u\left(t_{k}, x_{i-1}\right)}{(\Delta x)^{2}}+f\left(t_{k, x_{i}}\right)-\frac{(\Delta x)^{2}}{12} \frac{\partial^{4} u\left(t, \xi_{i}\right)}{\partial x^{4}}
\end{aligned}
$$

where we assume that:

$$
H(\alpha)=c(\alpha) \frac{\partial^{\alpha} u(t, x)}{\partial t^{\alpha}} \in C^{2}([0,1]), v \in(0,1) \text { and } \xi_{i} \in\left(x_{i-1}, x_{i+1}\right) .
$$

Then, from (37), it follows:

$$
L_{1}\left(u\left(t_{k,}, x_{i}\right)\right)=L_{2}\left(u\left(t_{k,}, x_{i}\right)\right)+f\left(t_{k}, x_{i}\right)+R_{i}^{k}, i=1, \ldots, K-1, k=1, \ldots, n,
$$

where:

$$
R_{i}^{k}=h \sum_{j=1}^{N} c\left(\alpha_{j}\right)\left(\delta_{t}^{\alpha_{j}} u\left(t_{k}, x_{i}\right)-\frac{\partial^{\alpha_{j}} u\left(t_{k}, x_{i}\right)}{\partial t^{\alpha_{j}}}\right)-\frac{h^{2}}{24} H^{\prime \prime}(v)-\frac{(\Delta x)^{2}}{12} \frac{\partial^{4} u\left(t, \xi_{i}\right)}{\partial x^{4}} .
$$

We easily see that defining:

$$
e_{i}^{k}=u\left(t_{k,}, x_{i}\right)-U_{i}^{k}, i=1, \ldots, K-1, k=1, \ldots, n,
$$

we have:

$$
\begin{cases}e_{i}^{0}=0, & i=1, \ldots, K-1 \\ L_{1}\left(e_{i}^{k}\right)=L_{2}\left(e_{i}^{k-1}\right)+R_{i}^{k}, & i=1, \ldots, K-1, k=1, \ldots, n .\end{cases}
$$

We start with some auxiliary lemmas.

Lemma 1. If the solution of (1) is such that the derivative in order to the time variable $t$ satisfies (4) is of class $C^{4}$ with respect to the variable $x$ and the function $H(\alpha)=c(\alpha) \frac{\partial^{\alpha} u(t, x)}{\partial t^{\alpha}} \in C^{2}([0,1])$, then there exists a positive constant $C$ independent of $n, N$, and $K$ such that:

$$
\left\|R^{k}\right\|_{\infty} \leq C\left(n^{-\mu} h \sum_{j=1}^{N} c\left(\alpha_{j}\right) t_{k}^{-\alpha_{j}}+h^{2}+(\Delta x)^{2}\right),
$$

where $\mu=\min \{a \beta, 1+h / 2\}$ and $a$ is defined as in Proposition 2.

Proof. Under the regularity assumptions on the solution (with respect to $x$ and $\alpha$ ), the result is straightforward since:

$$
\begin{aligned}
& \left|h \sum_{j=1}^{N} c\left(\alpha_{j}\right)\left(\delta_{t}^{\alpha_{j}} u\left(t_{k}, x_{i}\right)-\frac{\partial^{\alpha_{j}} u\left(t_{k}, x_{i}\right)}{\partial t^{\alpha_{j}}}\right)\right| \\
& \leq C h \sum_{j=1}^{N} c\left(\alpha_{j}\right) t_{k}^{-\alpha_{j}} n^{-\gamma_{j}} \text { with } \gamma_{j}=\min \left\{a \beta, 2-\alpha_{j}\right\} \\
& \leq C h \sum_{j=1}^{N} c\left(\alpha_{j}\right) t_{k}^{-\alpha_{j}} n^{-\gamma_{N}} \text { with } \gamma_{N}=\min \left\{a \beta, 2-\alpha_{N}\right\} \\
& \leq C n^{-\mu} h \sum_{j=1}^{N} c\left(\alpha_{j}\right) t_{k}^{-\alpha_{j}}
\end{aligned}
$$


where $C$ is a positive constant. Attending to the definition of $R_{i}^{k}$ (see (38)) follows (39).

Finally, we present the main result concerning the convergence of the numerical scheme (21)-(23).

Theorem 2. Assume that the solution of (1) is such that the derivative in order to the time variable $t$ satisfies (4), is of class $C^{4}$ with respect to the variable $x$ and the function $H(\alpha)=c(\alpha) \frac{\partial^{\alpha} u(t, x)}{\partial t^{\alpha}} \in$ $C^{2}([0,1])$.

Then, there exists a positive constant $C$ not depending on $n, N$, and $K$ such that:

$$
\left\|e^{k}\right\|_{\infty} \leq C\left(n^{-\mu}+(\Delta x)^{2}+h^{2}\right), \quad k=1,2, \ldots, n,
$$

where $\mu=\min \{a \beta, 1+h / 2\}$ with $a=r$ for the graded mesh (5), $a=2$ for the mesh (10), $a=3$ for the mesh (11), $a=4$ for the mesh (12), and $a=5$ for the mesh (13).

Proof. We prove this result by induction in $k$.

For $k=1$ :

$$
\begin{aligned}
& \Lambda\left(h, \tau_{0}\right)\left\|e^{1}\right\|_{\infty} \leq\left|L_{1}\left(e_{p}^{1}\right)\right|=\left|L_{2}\left(e_{p}^{0}\right)+R_{p}^{1}\right| \\
& \leq\left|L_{2}\left(e_{p}^{0}\right)\right|+\left\|R^{1}\right\|_{\infty} \\
& \leq\left|h \sum_{j=1}^{N} c\left(\alpha_{j}\right) \tau_{0}^{-1} a_{0,1}^{\alpha_{j}} e_{p}^{0}\right|+C\left(n^{-\mu} h \sum_{j=1}^{N} c\left(\alpha_{j}\right) t_{1}^{-\alpha_{j}}+h^{2}+(\Delta x)^{2}\right) \\
& =C\left(n^{-\mu} h \sum_{j=1}^{N} c\left(\alpha_{j}\right) t_{1}^{-\alpha_{j}}+h^{2}+(\Delta x)^{2}\right) \text {. }
\end{aligned}
$$

Then:

$$
\left\|e^{1}\right\|_{\infty} \leq \frac{C\left(n^{-\mu} h \sum_{j=1}^{N} c\left(\alpha_{j}\right) t_{1}^{-\alpha_{j}}+h^{2}+(\Delta x)^{2}\right)}{h \sum_{j=1}^{N} c\left(\alpha_{j}\right) \tau_{0}^{-1} a_{0,1}^{\alpha_{j}}}=\frac{C\left(n^{-\mu} h \sum_{j=1}^{N} c\left(\alpha_{j}\right) t_{1}^{-\alpha_{j}}+h^{2}+(\Delta x)^{2}\right)}{h \sum_{j=1}^{N} c\left(\alpha_{j}\right) \frac{t_{1}^{-\alpha_{j}}}{\Gamma\left(2-\alpha_{j}\right)}} .
$$

Since:

$$
\frac{t_{1}^{-\alpha_{j}}}{\Gamma\left(2-\alpha_{j}\right)} \geq T^{-\alpha_{j}} \geq \min \left\{T^{-\alpha_{1}}, T^{-\alpha_{N}}\right\} \geq C,
$$

and:

$$
h \sum_{j=1}^{N} c\left(\alpha_{j}\right) t_{1}^{-\alpha_{j}} \geq C h \sum_{j=1}^{N} c\left(\alpha_{j}\right)=C\left(\int_{0}^{1} c(\alpha) d \alpha+O\left(h^{2}\right)\right) \geq C,
$$

with $C$ positive constants, the result follows for $k=1$.

Now, suppose that:

$$
\left\|e^{\ell}\right\|_{\infty} \leq C\left(n^{-\mu}+(\Delta x)^{2}+h^{2}\right), \quad \ell=1,2, \ldots, k-1,
$$

and let $p \in \mathbb{N}$ be such that $\left\|e^{k}\right\|_{\infty}=e_{p}^{k}$.

Similar to the case $k=1$, using the induction argument and taking into account Proposition 2 and Lemma 1, it follows: 


$$
\begin{aligned}
\Lambda\left(h, \tau_{k-1}\right)\left\|e^{k}\right\|_{\infty} \leq & \left|L_{1}\left(e_{p}^{k}\right)\right|=\left|L_{2}\left(e_{p}^{k-1}\right)+R_{p}^{k}\right| \\
& \leq\left|L_{2}\left(e_{p}^{k-1}\right)\right|+\left\|R^{k}\right\|_{\infty} \\
& \leq\left|h \sum_{j=1}^{N} c\left(\alpha_{j}\right) \sum_{m=0}^{k-2}\left(\tau_{m+1}^{-1} a_{m+1, k}^{\alpha_{j}}-\tau_{m}^{-1} a_{m, k}^{\alpha_{j}}\right) e_{p}^{m+1}\right| \\
& +C\left(n^{-\mu} h \sum_{j=1}^{N} c\left(\alpha_{j}\right) t_{k}^{-\alpha_{j}}+h^{2}+(\Delta x)^{2}\right) \\
& \leq C\left(n^{-\mu} h+h^{2}+(\Delta x)^{2}\right) h \sum_{j=1}^{N} c\left(\alpha_{j}\right)\left(\tau_{k-1}^{-1} a_{k-1, k}^{\alpha_{j}}-\tau_{0}^{-1} a_{0, k}^{\alpha_{j}}\right) \\
& +C\left(n^{-\mu} h \sum_{j=1}^{N} c\left(\alpha_{j}\right) t_{k}^{-\alpha_{j}}+h^{2}+(\Delta x)^{2}\right) .
\end{aligned}
$$

Since $\tau_{k-1}^{-1} a_{k-1, k}^{\alpha_{j}}-\tau_{0}^{-1} a_{0, k}^{\alpha_{j}}<\tau_{k-1}^{-1} a_{k-1, k}^{\alpha_{j}}$, then from the inequality above, we obtain:

$$
\left\|e^{k}\right\|_{\infty} \leq C\left(n^{-\mu} h+h^{2}+(\Delta x)^{2}\right)+C \frac{\left(n^{-\mu} h \sum_{j=1}^{N} c\left(\alpha_{j}\right) t_{k}^{-\alpha_{j}}+h^{2}+(\Delta x)^{2}\right)}{h \sum_{j=1}^{N} c\left(\alpha_{j}\right) \tau_{k-1}^{-1} a_{k-1, k}^{\alpha_{j}}} .
$$

Note that:

$$
\tau_{k-1}^{-1} a_{k-1, k}^{\alpha_{j}}=\tau_{k-1}^{-1} \frac{\left(t_{k}-t_{k-1}\right)^{1-\alpha_{j}}}{\Gamma\left(2-\alpha_{j}\right)}=\frac{\tau_{k-1}^{-\alpha_{j}}}{\Gamma\left(2-\alpha_{j}\right)}>t_{k}^{-\alpha_{j}}
$$

for which, from (41), we obtain:

$$
\left\|e^{k}\right\|_{\infty} \leq C\left(n^{-\mu}+h^{2}+(\Delta x)^{2}\right)+C n^{-\mu}+C \frac{h^{2}+(\Delta x)^{2}}{h \sum_{j=1}^{N} c\left(\alpha_{j}\right) t_{k}^{-\alpha_{j}}} .
$$

Similar to the case $k=1$, we prove that:

$$
h \sum_{j=1}^{N} c\left(\alpha_{j}\right) t_{k}^{-\alpha_{j}} \geq C\left(\int_{0}^{1} c(\alpha) d \alpha+O\left(h^{2}\right)\right) \geq C .
$$

Thus, from (42), we have:

$$
\left\|e^{k}\right\|_{\infty} \leq C\left(n^{-\mu}+h^{2}+(\Delta x)^{2}\right) .
$$

Thus, the proof is complete.

\section{Numerical Results and Discussion}

We consider the numerical solution of a DODE of type (1) where $c(\alpha)=\frac{\Gamma(\beta-\alpha+1)}{\Gamma(\beta+1)}$, $u(t, x)=t^{\beta} \sin (x), \quad t>0, x \in[0, \pi]$, and $\beta \in(0,1)$.

In Tables $1-3$, we list the maximum of the absolute errors $\left(\|e\|_{\infty}\right)$ and experimental orders of convergence (EOCs) obtained with the numerical method (21)-(23) on uniform, graded, and nonuniform meshes defined by (10)-(13) and with different stepsizes (with no restrictions because the numerical scheme is unconditionally stable (see Theorem 1)). 
Table 1. $\beta=1 / 3$ : maximum of the errors and experimental convergence orders for a uniform mesh, a graded mesh, and Meshes $1,2,3$, and 4 .

\begin{tabular}{|c|c|c|c|c|c|c|c|c|c|c|c|c|c|c|c|c|c|c|c|}
\hline \multirow{2}{*}{$N=K$} & \multirow[b]{2}{*}{$n$} & \multicolumn{3}{|c|}{ Uniform Mesh } & \multicolumn{3}{|c|}{ Graded Mesh-r $r=4$} & \multicolumn{3}{|c|}{ Mesh 1} & \multicolumn{3}{|c|}{ Mesh 2} & \multicolumn{3}{|c|}{ Mesh 3} & \multicolumn{3}{|c|}{ Mesh 4} \\
\hline & & $\|e\|_{\infty}$ & $E O C_{t}$ & $E O C_{x}$ & $\|e\|_{\infty}$ & $E O C_{t}$ & $E O C_{x}$ & $\|e\|_{\infty}$ & $E O C_{t}$ & $E O C_{x}$ & $\|e\|_{\infty}$ & $E O C_{t}$ & $E O C_{x}$ & $\|e\|_{\infty}$ & $E O C_{t}$ & $E O C_{x}$ & $\|e\|_{\infty}$ & $E O C_{t}$ & $E O C_{x}$ \\
\hline 2 & 4 & $1.67 \times 10^{-1}$ & - & - & $6.67 \times 10^{-2}$ & - & - & $1.46 \times 10^{-1}$ & - & - & $1.07 \times 10^{-1}$ & - & - & $7.13 \times 10^{-2}$ & - & - & $6.00 \times 10^{-2}$ & - & - \\
\hline 4 & 16 & $1.66 \times 10^{-1}$ & 0.00 & 0.01 & $2.79 \times 10^{-2}$ & 0.63 & 1.26 & $9.28 \times 10^{-2}$ & 0.33 & 0.65 & $4.69 \times 10^{-2}$ & 0.60 & 1.19 & $2.87 \times 10^{-2}$ & 0.66 & 1.31 & $2.30 \times 10^{-2}$ & 0.69 & 1.38 \\
\hline 16 & 256 & $8.60 \times 10^{-2}$ & 0.27 & 0.55 & $2.66 \times 10^{-3}$ & 0.89 & 1.78 & $2.10 \times 10^{-2}$ & 0.56 & 1.12 & $6.39 \times 10^{-3}$ & 0.76 & 1.51 & $2.69 \times 10^{-3}$ & 0.89 & 1.79 & $1.54 \times 10^{-3}$ & 1.03 & 2.05 \\
\hline 32 & 1024 & $5.66 \times 10^{-3}$ & 0.30 & 0.60 & $7.16 \times 10^{-4}$ & 0.95 & 1.89 & $9.25 \times 10^{-3}$ & 0.59 & 1.19 & $2.07 \times 10^{-3}$ & 0.81 & 1.62 & $7.20 \times 10^{-4}$ & 0.95 & 1.90 & $3.50 \times 10^{-4}$ & 1.07 & 2.14 \\
\hline
\end{tabular}

Table 2. $\beta=1 / 2$ : maximum of the errors and experimental convergence orders for a uniform mesh, a graded mesh, and Meshes $1,2,3$, and 4 .

\begin{tabular}{|c|c|c|c|c|c|c|c|c|c|c|c|c|c|c|c|c|c|c|c|}
\hline \multirow[b]{2}{*}{$N=K$} & \multirow[b]{2}{*}{$n$} & \multicolumn{3}{|c|}{ Uniform Mesh } & \multicolumn{3}{|c|}{ Graded Mesh $-r=3$} & \multicolumn{3}{|c|}{ Mesh 1} & \multicolumn{3}{|c|}{ Mesh 2} & \multicolumn{3}{|c|}{ Mesh 3} & \multicolumn{3}{|c|}{ Mesh 4} \\
\hline & & $\|e\|_{\infty}$ & $E O C_{t}$ & $E O C_{x}$ & $\|e\|_{\infty}$ & $E O C_{t}$ & $E O C_{x}$ & $\|e\|_{\infty}$ & $E O C_{t}$ & $E O C_{x}$ & $\|e\|_{\infty}$ & $E O C_{t}$ & $E O C_{x}$ & $\|e\|_{\infty}$ & $E O C_{t}$ & $E O C_{x}$ & $\|e\|_{\infty}$ & $E O C_{t}$ & $E O C_{x}$ \\
\hline 2 & 4 & $7.87 \times 10^{-2}$ & - & - & $2.81 \times 10^{-2}$ & - & - & $6.13 \times 10^{-2}$ & - & - & $3.63 \times 10^{-2}$ & - & - & $2.46 \times 10^{-2}$ & - & - & $1.94 \times 10^{-2}$ & - & - \\
\hline 4 & 16 & $7.15 \times 10^{-2}$ & 0.07 & 0.14 & $1.17 \times 10^{-2}$ & 0.63 & 1.26 & $2.85 \times 10^{-2}$ & 0.55 & 1.11 & $1.23 \times 10^{-2}$ & 0.78 & 1.56 & $8.43 \times 10^{-3}$ & 0.77 & 1.54 & $8.20 \times 10^{-3}$ & 0.62 & 1.24 \\
\hline 8 & 64 & $4.46 \times 10^{-2}$ & 0.34 & 0.68 & $3.80 \times 10^{-3}$ & 0.81 & 1.62 & $1.05 \times 10^{-2}$ & 0.72 & 1.43 & $3.81 \times 10^{-3}$ & 0.85 & 1.70 & $3.37 \times 10^{-3}$ & 0.66 & 1.32 & $2.83 \times 10^{-3}$ & 0.77 & 1.53 \\
\hline 16 & 256 & $2.47 \times 10^{-2}$ & 0.43 & 0.85 & $1.07 \times 10^{-3}$ & 0.91 & 1.83 & $3.51 \times 10^{-3}$ & 0.79 & 1.58 & $1.07 \times 10^{-3}$ & 0.92 & 1.83 & $9.99 \times 10^{-4}$ & 0.88 & 1.76 & $9.02 \times 10^{-4}$ & 0.83 & 1.65 \\
\hline 32 & 1024 & $1.35 \times 10^{-2}$ & 0.44 & 0.88 & $2.84 \times 10^{-4}$ & 0.96 & 1.91 & $1.09 \times 10^{-3}$ & 0.84 & 1.69 & $2.84 \times 10^{-4}$ & 0.96 & 1.91 & $2.72 \times 10^{-4}$ & 0.94 & 1.88 & $2.52 \times 10^{-4}$ & 0.92 & 1.84 \\
\hline
\end{tabular}

Table 3. $\beta=2 / 3$ : maximum of the errors and experimental convergence orders for a uniform mesh, a graded mesh, and Meshes 1, 2, 3, and 4.

\begin{tabular}{|c|c|c|c|c|c|c|c|c|c|c|c|c|c|c|c|c|c|c|c|}
\hline \multirow{2}{*}{$N=K$} & \multirow{2}{*}{$n$} & \multicolumn{3}{|c|}{ Uniform Mesh } & \multicolumn{3}{|c|}{ Graded Mesh-r $=2$} & \multicolumn{3}{|c|}{ Mesh 1} & \multicolumn{3}{|c|}{ Mesh 2} & \multicolumn{3}{|c|}{ Mesh 3} & \multicolumn{3}{|c|}{ Mesh 4} \\
\hline & & $\|e\|_{\infty}$ & $E O C_{t}$ & $E O C_{x}$ & $\|e\|_{\infty}$ & $E O C_{t}$ & $E O C_{x}$ & $\|e\|_{\infty}$ & $E O C_{t}$ & $E O C_{x}$ & $\|e\|_{\infty}$ & $E O C_{t}$ & $E O C_{x}$ & $\|e\|_{\infty}$ & $E O C_{t}$ & $E O C_{x}$ & $\|e\|_{\infty}$ & $E O C_{t}$ & $E O C_{x}$ \\
\hline 2 & 4 & $4.49 \times 10^{-2}$ & - & - & $3.05 \times 10^{-2}$ & - & - & $3.42 \times 10^{-2}$ & - & - & $2.17 \times 10^{-2}$ & - & - & $1.10 \times 10^{-2}$ & - & - & $4.49 \times 10^{-2}$ & - & - \\
\hline 4 & 16 & $2.68 \times 10^{-2}$ & 0.37 & 0.74 & $1.68 \times 10^{-2}$ & 0.43 & 0.86 & $1.69 \times 10^{-2}$ & 0.51 & 1.02 & $1.47 \times 10^{-2}$ & 0.28 & 0.57 & $1.21 \times 10^{-3}$ & $(*)$ & $(*)$ & $9.50 \times 10^{-3}$ & 4.44 & 8.88 \\
\hline 8 & 64 & $1.40 \times 10^{-2}$ & 0.47 & 0.94 & $4.95 \times 10^{-3}$ & 0.88 & 1.76 & $4.95 \times 10^{-3}$ & 0.89 & 1.77 & $4.67 \times 10^{-3}$ & 0.83 & 1.66 & $4.28 \times 10^{-3}$ & 0.75 & 1.50 & $3.85 \times 10^{-3}$ & 0.65 & 1.30 \\
\hline 16 & 256 & $6.57 \times 10^{-3}$ & 0.55 & 1.10 & $1.31 \times 10^{-3}$ & 0.96 & 1.92 & $1.31 \times 10^{-3}$ & 0.96 & 1.92 & $1.26 \times 10^{-3}$ & 0.94 & 1.88 & $1.20 \times 10^{-3}$ & 0.92 & 1.84 & $1.12 \times 10^{-3}$ & 0.89 & 1.78 \\
\hline 32 & 1024 & $2.91 \times 10^{-3}$ & 0.59 & 1.17 & $3.35 \times 10^{-4}$ & 0.98 & 1.96 & $3.35 \times 10-4$ & 0.98 & 1.96 & $3.28 \times 10^{-4}$ & 0.97 & 1.95 & $3.15 \times 10^{-4}$ & 0.96 & 1.93 & $3.01 \times 10^{-4}$ & 0.95 & 1.90 \\
\hline
\end{tabular}


To test the robustness of the numerical method and to assess the theoretical convergence orders derived in the previous section, we considered three different $\beta$ values of $1 / 3,1 / 2,2 / 3$ ( $\beta=1 / 3$ leads to a strong singularity and $\beta=2 / 3$ to a weaker singularity).

A more careful analysis of Tables $1-3$ reveals that the time convergence order is quite low for the uniform mesh (for all $\beta$ values considered). As expected, decreasing the strength of the singularity (increasing $\beta$ ), the convergence order also increases, being 0.3 for $\beta=1 / 3$ and 0.6 for $\beta=2 / 3$.

Regarding graded meshes, we considered a grading that resulted in the optimal order, for each of the $\beta$ values. For $\beta=1 / 3$, the convergence order was slightly smaller when compared to the other two cases, but within the expected optimal order of convergence $(\approx 1)$. The disadvantage of the graded mesh is that in order to obtain the optimal order of convergence, one must know, a priori, the analytical solution (more precisely, the order of the singularity). This makes no sense since the objective is to obtain a numerical solution, because the analytical solution is not known. A possible solution is to perform a large number of simulations considering different $r$ values, until the optimal (or near-optimal) convergence is obtained. This method is time consuming, especially for distributedorder derivatives.

This being said, we propose a new methodology to solve this problem, based on the use of nonuniform meshes. These meshes allow one to obtain the optimal (or near-optimal) convergence order without knowing the analytical solution, a priori. We analyzed the numerical results obtained for the four nonuniform meshes presented in the Introduction, and the results are now compared with the theoretical expected order of convergence for each mesh and $\beta$ value.

Note that, in the previous section, we concluded that,

$$
\left\|e^{k}\right\|_{\infty} \leq C\left(n^{-\mu}+(\Delta x)^{2}+h^{2}\right), k=0,1,2, \ldots, n-1
$$

where $a=2$ for the mesh (10) (Mesh 1), $a=3$ for the mesh (11) (Mesh 2), $a=4$ for the mesh (12) (Mesh 3), and $a=5$ for the mesh (13) (Mesh 4). Furthermore, we have that $\mu=\min \{a \beta, 1+h / 2\}$ :

- $\quad$ For Mesh 1 and $\beta=1 / 3$, we have that $\mu=\min \{2 / 3,1+h / 2\}=2 / 3$;

- $\quad$ For Mesh 2 and $\beta=1 / 3$, we have that $\mu=\min \{1,1+h / 2\}=1$;

- For Mesh 3 and $\beta=1 / 3$, we have that $\mu=\min \{4 / 3,1+h / 2\} \approx 1$ (since $h \rightarrow 0$ );

- For Mesh 3 and $\beta=1 / 3$, we have that $\mu=\min \{5 / 3,1+h / 2\} \approx 1$ (since $h \rightarrow 0$ ).

Therefore, we were expecting a better convergence order (in time) for Meshes 3 and 4, which is the case (see Table 1). We obtained a time convergence order of 0.95 and 1.07 for Meshes 3 and 4. For Mesh 1, we obtained a time convergence order of 0.59 , which is in accordance with the expected theoretical convergence rate of $2 / 3$ :

- $\quad$ For Mesh 1 and $\beta=1 / 2$, we have that $\mu=\min \{1,1+h / 2\}=1$;

- For Mesh 2 and $\beta=1 / 2$, we have that $\mu=\min \{3 / 2,1+h / 2\} \approx 1$ (since $h \rightarrow 0$ );

- For Mesh 3 and $\beta=1 / 2$, we have that $\mu=\min \{2,1+h / 2\} \approx 1$ (since $h \rightarrow 0$ );

- For Mesh 3 and $\beta=1 / 2$, we have that $\mu=\min \{5 / 2,1+h / 2\} \approx 1$ (since $h \rightarrow 0$ ).

In this case, we were expecting a good convergence order (in time) for all meshes, which is the case (see Table 2). The convergence orders were slightly higher for Meshes 2, 3 , and 4 , because in these meshes, there is room for a convergence order higher than one. The best convergence order was obtained for Mesh 2:

- For Mesh 1 and $\beta=2 / 3$, we have that $\mu=\min \{4 / 3,1+h / 2\} \approx 1$ (since $h \rightarrow 0$ );

- For Mesh 2 and $\beta=2 / 3$, we have that $\mu=\min \{2,1+h / 2\} \approx 1$ (since $h \rightarrow 0$ );

- $\quad$ For Mesh 3 and $\beta=2 / 3$, we have that $\mu=\min \{8 / 3,1+h / 2\} \approx 1$ (since $h \rightarrow 0$ );

- $\quad$ For Mesh 3 and $\beta=2 / 3$, we have that $\mu=\min \{10 / 3,1+h / 2\} \approx 1$ (since $h \rightarrow 0$ ). 
In this case, we have a weaker singularity, and we were expecting a good convergence order (in time) for all meshes, which is the case (see Table 3). Furthermore, as expected, we obtained the best convergence order for Mesh 1.

By looking at the global results presented in Tables 1-3, we concluded that Meshes 3 and 4 provide an excellent convergence order in time, for all $\beta$ values considered, thus being a good alternative to graded meshes.

\section{Conclusions}

A similar analysis as the one carried out in [20] allowed us to conclude that the numerical scheme presented in this work is unconditionally stable and convergent with accuracy $O\left(N^{-2}+K^{-2}+n^{-1}\right)$ for sufficiently smooth solutions.

For nonsmooth solutions, we showed that finite difference schemes on uniform grids provide results with the loss of the convergence order. To overcome this problem, we proposed an alternative numerical method based on time nonuniform meshes. We proved the stability and convergence of the numerical method, resulting in a convergence order $O\left(n^{-\mu}+(\Delta x)^{2}+h^{2}\right)$ (with $a=r$ for graded meshes with grading exponent $r \geq 1, a=2$ for Mesh 1, $a=3$ for Mesh 2, $a=4$ for Mesh 3, and $a=5$ for Mesh 4, being $\mu=$ $\min \{a \beta, 1+h / 2\})$.

The use of nonuniform meshes improved the results (as illustrated in the numerical results above), allowing us to recover the optimal convergence order (in time) for different singularities, and moreover, nonuniform meshes of the type (9) can be a good alternative to graded meshes.

A topic for further investigation is the smoothness properties of typical solutions of this kind of problems, at least for some particular choices of the weighting function $c(\cdot)$.

Author Contributions: Conceptualization, M.L.M.; methodology, M.L.M.; software, M.L.M., M.R., and L.L.F.; validation, M.L.M., M.R., and L.L.F.; formal analysis, M.L.M.; investigation, M.L.M., M.R., and L.L.F.; writing—original draft preparation, M.L.M.; writing—review and editing, M.L.M., M.R., and L.L.F.; supervision, M.L.M.; funding acquisition, M.L.M., M.R., and L.L.F. All authors read and agreed to the published version of the manuscript.

Funding: This research was funded by Fundação para a Ciência e Tecnologia within Projects UIDB/04621/2020 and UIDP/04621/2020, Project UIDB/00297/2020 (Center for Mathematics and Applications), and through Projects UIDB/00013/2020 and UIDP/00013/2020 (CMAT-Centre of Mathematics-University of Minho).

Institutional Review Board Statement: Not applicable.

Informed Consent Statement: Not applicable.

Acknowledgments: The authors acknowledge the support of the Center for Mathematics and Applications (CMA) - FCT-NOVA, Center for Computational and Stochastic Mathematics, Instituto Superior Técnico, and CMAT-Centre of Mathematics-University of Minho. The first author acknowledges Fundação para a Ciência e Tecnologia (Portuguese Foundation for Science and Technology) within Projects UIDB/04621/2020 and UIDP/04621/2020. The second author acknowledges the Fundação para a Ciência e a Tecnologia through Project UIDB/00297/2020 (Centro de Matemática e Aplicações). The third author acknowledges the funding by Fundação para a Ciência e Tecnologia through Projects UIDB/00013/2020 and UIDP/00013/2020.

Conflicts of Interest: The authors declare no conflict of interest.

\section{Abbreviations}

The following abbreviations are used in this manuscript:

EOC Experimental order of convergence 


\section{References}

1. Caputo, M. Elasticità e Dissipazione; Zanichelli: Bologna, Italy, 1969.

2. Caputo, M. Mean fractional-order-derivatives differential equations and filters. Ann. Dell Univ. Ferrara. Nuova Serie. Sez. VII 1995, 41,73-84. [CrossRef]

3. Caputo, M. Distributed order differential equations modelling dielectric induction and diffusion. Fract. Calc. Appl. Anal. 2001, 4, 421-442. [CrossRef]

4. Bagley, R.L.; Torvik, P.J. On the existence of the order domain and the solution of distributed order equations-Part I. Int. J. Appl. Math. 2000, 2, 865-882. [CrossRef]

5. Bagley, R.L.; Torvik, P.J. On the existence of the order domain and the solution of distributed order equations-Part II. Int. J. Appl. Math. 2000, 2, 965-987. [CrossRef]

6. Gorenflo, R.; Luchko, Y.; Stojanovic, M. Fundamental solution of a distributed order time-fractional diffusion-wave equation as probability density. Fract. Calc. Appl. Anal. 2013, 16, 297-316. [CrossRef]

7. Mainardi, F.; Pagnini, G.; Mura, A.; Gorenflo, R. Time-fractional diffusion of distributed order. J. Vib. Control 2008, 14, 1267-1290. [CrossRef]

8. Chechkin, A.V.; Gorenflo, R.; Sokolov, I.M. Retarding subdiffusion and accelerating superdiffusion governed by distributed-order fractional diffusion equations. Phys. Rev. E 2002, 66, 046129. [CrossRef]

9. Diethelm, K.; Ford, N.J. Numerical solution methods for distributed order differential equations. Fract. Calc. Appl. Anal. 2001, 4, 531-542.

10. Ford, N.J.; Morgado, M.L. Distributed order equations as boundary value problems. Comput. Math. Appl. 2012, 64, $2973-2981$.

11. Morgado, M.L.; Rebelo, M. Numerical approximation of distributed order reaction-diffusion equations. J. Comput. Appl. Math. 2015, 275, 216-227. [CrossRef]

12. Ye, H.; Liu, F.; Anh, V. Compact difference scheme for distributed-order time-fractional diffusion-wave equation on bounded domains. J. Computat. Phys. 2015, 298, 652-660.

13. Wang, X.; Liu, F.; Chen, X. Novel Second-Order Accurate Implicit Numerical Methods for the Riesz Space Distributed-Order Advection-Dispersion Equations. Adv. Math. Phys. 2015, 2015, 590435.

14. Jin, B.; Lazarov, R.; Sheen, D.; Zhou, Z. Error estimates for approximations of distributed order time fractional diffusion with nonsmooth data. Frac. Calc. Appl. Anal. 2016, 19, 69-93.

15. Morgado, M.L.; Rebelo, M.; Ferrás, L.L.; Ford, N.J. Numerical solution for diffusion equations with distributed order in time using a Chebyshev collocation method. Appl. Numer. Math. 2017, 114, 108-123.

16. Stynes, M.; O'Riordan, E.; Gracia, J.L. Error analysis of a finite difference method on graded meshes for a time-fractional diffusion equation. SIAM J. Numer. Anal. 2017, 55, 1057-1079.

17. Changpin, L.; Qian Y.; Chen A. Finite difference methods with nonuniform meshes for nonlinear fractional differential equations. J. Comput. Phys. 2016, 316, 614-631.

18. Morgado, M.L.; Morgado, L.F. Modeling transient currents in time-of-flight experiments with tempered time-fractional diffusion equations. Progr. Fract. Differ. Appl. 2020, 6, 43-53.

19. Kopteva, N. Error analysis of the L1 method on graded and uniform meshes for a fractional-derivative problem in two and three dimensions. Math. Comput. 2019, 88, 2135-2155.

20. Ford, N.J.; Morgado, M.L.; Rebelo, M. An implicit finite difference approximation for the solution of the diffusion equation with distributed order in time. Electron. Trans. Numer. Anal. 2015, 44, 289-305. 